\title{
Massive hemoptysis in a child: An unusual presentation of hydatid cyst
}

\section{Doddaiah Narayanappa, Hassan Sreenivasamurthy Rajani, Mathod Ganeshrao Anilkumar, Sangaraju Mamatha \\ Department of Pediatrics, JSS Medical College, JSS University, Mysore, Karnataka, India}

Address for the Correspondence: Dr. Rajani H.S, Department of Pediatrics, JSS Hospital, Mahatma Gandhi Road, Mysore, Karnataka, India. E-mail: drrajanihs@gmail.com

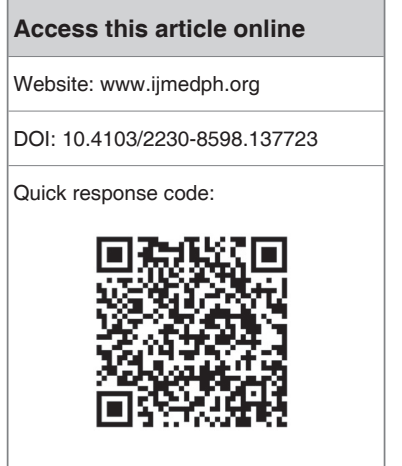

\begin{abstract}
A 10-year-old male child presented with recurrent hemoptysis since 1 month and a bout of massive hemoptysis on the previous day of admission. Chest radiography and CT scan revealed a soft tissue inflammatory mass in the right lower lobe. Emergency right lower lobectomy revealed a cavitatory lesion with membrane, communicating with bronchus. Histopathology confirmed the diagnosis of hydatid cyst.
\end{abstract}

Key words: Child, hemoptysis, hydatid cyst

\section{INTRODUCTION}

Human echinococcosis is a zoonotic infection caused by the tapeworm of the genus echinococcus. Hydatid cysts grow slowly and is rarely diagnosed during childhood or adolescence ${ }^{[1]}$ and is a rare cause of hemoptysis in children. ${ }^{[2]}$ Hereby, we are reporting a case of hydatid cyst lung presenting with massive hemoptysis in a child.

\section{CASE REPORT}

A 10-year-old male child was brought with history of 1 month productive cough with blood-streaked sputum initially which progressed to expectoration of frank blood around 100-150 ml per day on the previous day of admission to our hospital. He initially had cough with dark clotted blood followed by frothy bright red frank blood. No history of trauma, contact with tuberculosis, history suggestive of congenital heart disease, any contact with a pet dog or sheep was present. No history of any other bleeding manifestations.

On examination, he was afebrile with mild tachypnoea. He was pale with grade III clubbing. There was no icterus or significant lymphadenopathy. On respiratory system examination, normal vesicular breath sounds heard with impaired note and decreased breath sounds in the right interscapular and infrascapular region. There was no adventitious sounds or bronchial breathing noted. Per abdomen examination revealed soft hepatomegaly $3 \mathrm{cms}$ below right costal margin in mid-clavicular line. He had ejection systolic murmur in pulmonary area on cardiovascular system examination.

Investigations revealed hemoglobin of $6.6 \mathrm{gm}$, total count of 10,100 cells per cumm with $75 \%$ neutrophils, ESR of 15 and normal platelet count of 3.4 lakhs. PT and aPT'T were within normal limits.PBS showed normocytic normochromic anemia with relative neutrophilia. Chest X-ray revealed a round pneumonia in the right lower zone [Figure 1]. Differential diagnosis considered were lung abscess, round pneumonia, hydatid cyst lung, sequestrated lung with secondary infection, bronchogenic cyst, lymphoma, adenoma and histoplasma.

CT scan revealed rounded mildly enhancing heterogenous soft tissue density lesion involving the posterior basal segment of right lower lobe abutting pleura with few enlarged pretracheal, retrocaval, right hilar and subcarinal nodes with no pleural effusion and rest of the lung fields being normal suggestive of inflammatory lesion [Figure 2].

Ultrasound abdomen was done to rule out any hepatic hydatid cyst. Lower sections of CT scan also did not reveal any hepatic hydatid cyst.

He was started on antibiotics co-amoxyclav, Amikacin and Metronidazole. Blood transfusion was given. Child developed massive hemoptysis of 200-300 $\mathrm{ml}$ frank blood on the next day of admission. 
To prevent exsanguinations he was taken up for emergency right lower lobectomy. He received another two units of blood transfusion during intraoperative and postoperative period.

Histopathology revealed a lesion measuring $9 \times 7 \times 7 \mathrm{cms}$, weighing $170 \mathrm{gms}$. Cut section showed a cavity measuring $2.5 \times 2.0 \mathrm{cms}$, lined by a membranous structure and grey-white irregular surface [Figures 3 and 4]. Section from membranous fragment shows trilaminated wall suggestive of hydatid cyst. Dense neutrophilic infiltration with many hemosiderin-laden macrophages and foreign body giant cells, congested and dilated blood vessels were noted.

Child was started on albendazole in a dose of $15 \mathrm{mg} / \mathrm{kg} /$ day for 4 weeks. He had an uneventful postoperative recovery and was discharged on tenth postoperative day.

\section{DISCUSSION}

Hydatidosis caused by Echinococcus granulosus is an endemic parasitic disease in Mediterranean countries, North Africa, Spain, Greece, Turkey, Portugal, Middle East, Australia, New Zealand,

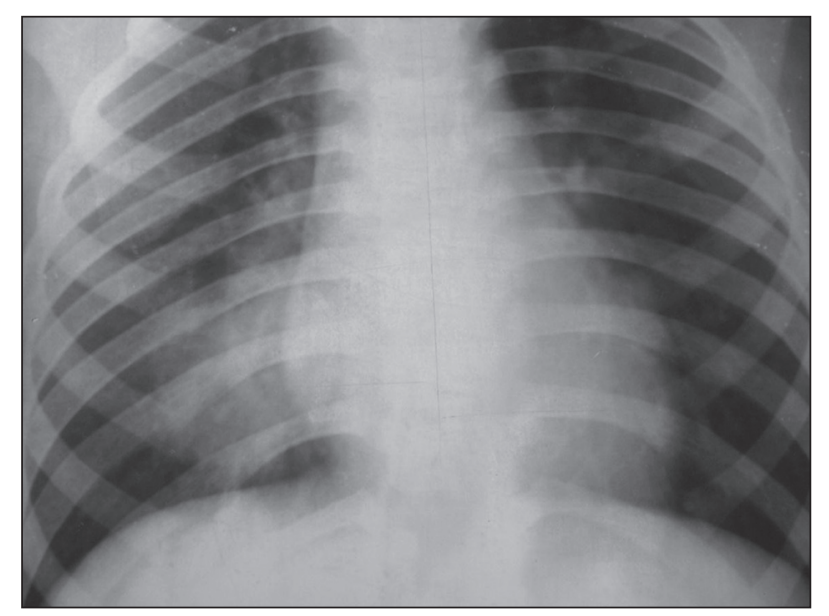

Figure 1: Chest $x$ ray revealed a round pneumonia in the right lower zone

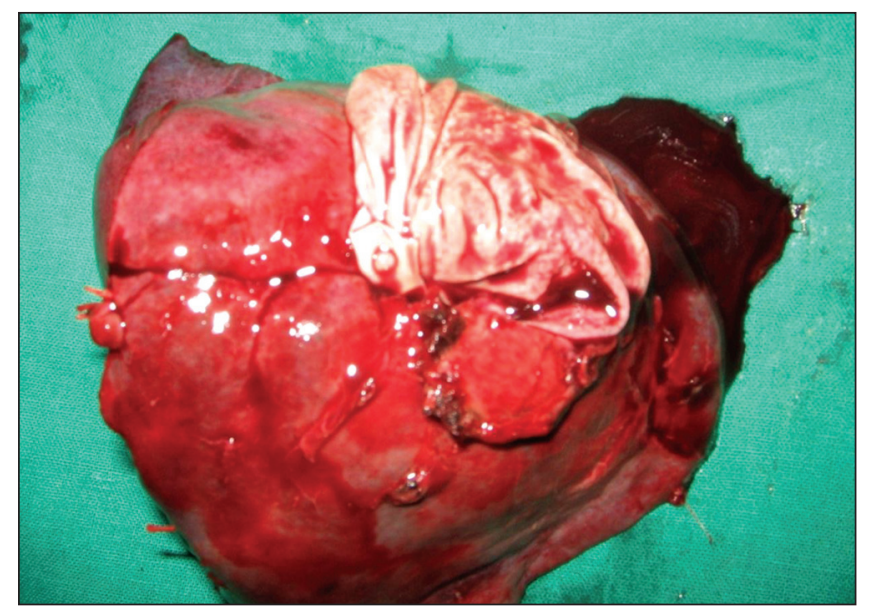

Figure 3: Cyst wall from the lesion
South America, Baltic areas, the Philippines, Northern China, and the Indian sub-continent. In India, it is reported from almost all parts of the country but higher incidence of the disease is reported from Tamil Nadu and Andhra Pradesh. ${ }^{[1]}$

There are four species of Taenia echinococcus: Echinococcus granulosus, Echinococcus multilocularis, Echinococcus oligartus, Echinococcus vogeli.

The definitive hosts are the red fox, arctic fox, dog and cat while the intermediate hosts are various members of the rodent family. Human hydatid disease is commonly caused by the parasite Echinococcus granulosus that has the dog as the definitive host and sheep as the intermediate host. Humans are accidental intermediate hosts. Human disease is rare but life threatening. ${ }^{[1]}$

Many hydatid cysts remain asymptomatic. Parasite load, the site, and the size of the cysts determine the degree of symptoms. A history of living in or visiting an endemic area, exposure to the parasite through the ingestion of foods or water contaminated by the feces of a definitive host must be determined.

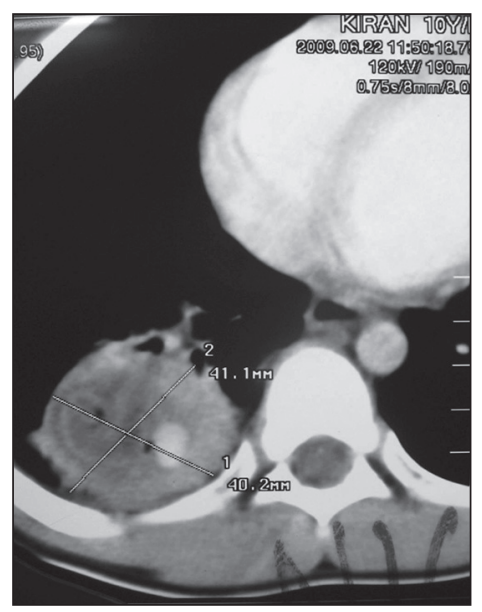

Figure 2: Ct scan: Small to moderate sized, rounded, mildly enhancing, heterogeneous soft tissue density lesion involving the posterior basal segment of right lower lobe, abutting the pleura

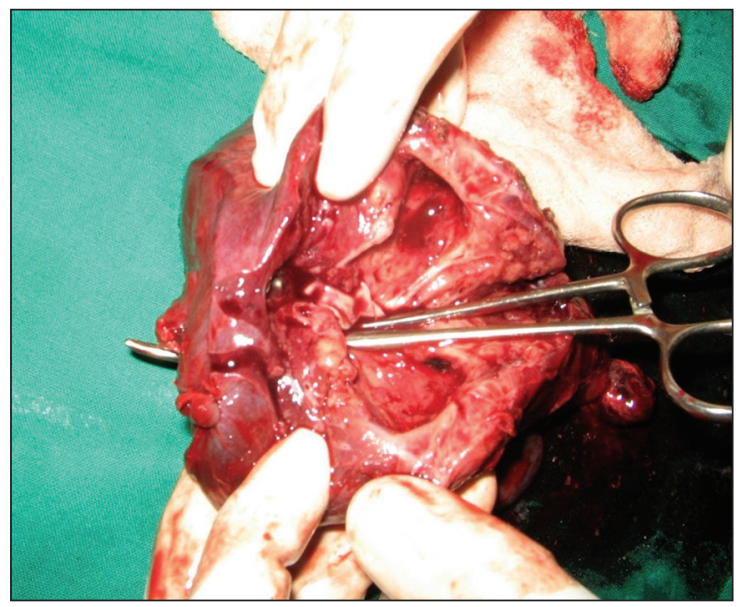

Figure 4: Lesion communicating with bronchus 
The common mode of infection is the unhygienic practice of consuming unwashed or improperly washed infected raw fruits and vegetables. Direct contact with infected dogs is also another means of contracting the disease, especially in children. ${ }^{[1]}$

In this child there was no history of contact with pets.

Frequency of location of hydatid disease in humans are liver (55-75\%) and lung (18-35\%). Other sites are peritoneal cavity $(1016 \%)$, kidney, spleen, uterus, retroperitoneum and peritoneum. ${ }^{[1]}$

Cough, chest pain and breathlessness are the most common presenting symptoms. In adult patients, in up to $70 \%$, hemoptysis is a presenting symptom but rare in pediatric patients. Hydatid cyst is a rare cause of hemoptysis in children. ${ }^{[2]}$ Common causes of pediatric hemoptysis include infection, foreign body aspiration, congenital heart disease, cystic fibrosis, pulmonary hemosiderosis, trauma, tracheostomy-related problems and fictitious hemoptysis. ${ }^{[2]}$

The mechanism of hemoptysis in pulmonary hydatid disease may be due to pressure erosion of a bronchus or obstruction with secondary bronchial infection or occasional rupture of cyst into the bronchus. This may in turn lead to further erosion into a branch arterial supply leading to hemoptysis. Hydatid cysts not only erode into bronchi, but also vascular structures including aorta causing massive hemoptysis. ${ }^{[2]}$

There are very few case reports of hydatid cyst presenting as hemoptysis in children. Bharati et al. ${ }^{[3]}$ have reported a 6 -year-old child with small amount of hemoptysis with diagnosis of pulmonary hydatid cyst in the left upper and lower lobes in addition to four cysts in the right lobe of liver. Singh S et al., ${ }^{[4]}$ have reported a 10 -year-old child with recurrent hemoptysis and a bout of massive hemoptysis with diagnosis of pulmonary hydatid cyst. The incidence of hemoptysis in children may be as high as 38\% as reported by Solak et al..$^{[5]}$

In our child, echinococcal cyst could have initially caused obstruction to branch bronchi leading to stasis and infection. Later erosion of branch bronchi and peripheral pulmonary artery might have resulted in massive hemoptysis.

CT scan has an accuracy of $98 \%$ and the sensitivity to demonstrate the daughter cysts. Classical radiological signs on CT scan are crescent sign, water lily sign (endocyst membrane floating on top of remaining fluid consequent to collapse of endocyst and partial evacuation of fluid), tiny bleb of air dissecting into the wall: Signet ring sign, air bubble sign (single or multiple small, rounded radiolucent areas with very sharp margins within solid media or pericystic areas due to dissection of air between the pericyst and parasitic membrane due to rupture or erosion of a bronchiole) but ruptured cysts may present with a wide variety of radiological appearances due to different combinations of collapsed membrane, air and fluid. ${ }^{[6]}$ Attenuation values in infected cysts are considerably higher than in unruptured ones producing solid appearance and causing diagnostic errors as in this case where CT scan revealed only mildly enhancing, heterogeneous soft tissue density lesion involving the posterior basal segment of right lower lobe.

Histopathology which revealed a trilaminated wall confirmed diagnosis of hydatid cyst in our case. The hydatid cyst has three layers: The outer pericyst, composed of modified host cells that form a dense and fibrous protective zone; the middle laminated membrane, which is acellular and allows the passage of nutrients; and the inner germinal layer, where the scolices (the larval stage of the parasite and the laminated membrane are produced).

The principles of surgical management of a pulmonary hydatid disease include complete evacuation of the cyst with preservation of normal lung parenchyma as much as possible ideally combined with medical management with benzimidazoles for 4 weeks. For cysts with abscesses or severe pulmonary destruction, segmentectomy, lobectomy or even pneumonectomy are required. ${ }^{[7]}$

In this case, child underwent emergency lobectomy to prevent exsanguinations and was discharged on oral albendazole for 4 weeks.

The pitfalls in diagnosis of hydatid disease in this child were there was no history of contact with pet dog or sheep rearing, the cysts grow slowly and a cyst is rarely diagnosed during childhood or adolescence, no peripheral blood eosinophilia, massive hemoptysis is not a common presentation in children, CT scan revealed a mildly enhancing lesion without classical signs.

In pediatric patients with life-threatening hemoptysis and an undiagnosed pulmonary mass that does not resemble hydatid disease of the lung, complicated hydatid cysts should be considered in the differential diagnosis.

\section{REFERENCES}

1. Dandan IS. Hydatid cysts. Available from: http://www.emedicine. medscape.com/article/178648-overview [Last accessed on 2008].

2. Gaude GS. Hemoptysis in Children. Indian Pediatr 2010;47:245-54.

3. Bharti S, Bharti B. Hydatid disease of lungs unusual cause of hemoptysis. Indian Pediatr 2002;39:1062-3.

4. Singh S, Vimesh P, Nadeem SA. Massive hemoptysis in children unusual presentation in pulmonary hydatid disease. Available from: http:// www.ctsnet.org/sections/clinicalresources/clinicalcases/article-10.html [Last accessed on 28/10/2013].

5. Solak H, Yenitzeri M, Yuksek T, Anil N, Goktogan T, Ceran S. The hydatid cyst of lung in children and results of surgical treatment. Thorac Cardiovasc Surg 1990;38:45-7.

6. Sharifi-Mood B, Fazaeli A, Izadi SH, Mokhtari S. Fifteen Years Experience with Pulmonary Hydatidosis in Zahedan, Iran. Iran J Parasitol 2007;2:7-11.

7. Wu MB, Zhang LW, Zhu H, Qian ZX. Surgical treatment for thoracic hydatidosis: Review of 1230 cases. Chin Med J (Engl) 2005;118:1665-7.

How to cite this article: Narayanappa D, Rajani HS, Anilkumar MG, Mamatha S. Massive hemoptysis in a child: An unusual presentation of hydatid cyst. Int J Med Public Health 2014;4:304-6.

Source of Support: Nil, Conflict of Interest: None declared. 